\title{
Perú 2017: un CASo EXTREMo De GobIERNo diVIDIDo
}

Peru 2017: An Extreme Case of Divided Government

\author{
MOISÉS ARCE \\ University of Missouri, Estados Unidos
}

\section{JOSÉ INCIO}

University of Pittsburgh, Estados Unidos

\begin{abstract}
RESUMEN
En el repertorio de frases populares peruanas, el dicho "se sufre pero se goza" puede resumir muy bien el 2017. Durante ese año los peruanos hemos tenido innumerables sin sabores en el nivel social, político y económico. El gobierno de turno, sin terminar de asentarse, tuvo que enfrentar los estragos del fenómeno del Niño, crisis ministeriales, pedidos de vacancia, entre otros, al tiempo que la economía se desaceleraba y la pobreza aumentaba. Sin embargo, creemos que para la mayoría de peruanos el 2017 también significa alegría, especialmente cuando recuerde el año con la agónica clasificación de la selección al Mundial de Futbol después de 36 años de ausencia. En este artículo tratamos resumir el contexto social y político en 2017, enfocándonos en el eje Ejecutivo-Legislativo que determinó los eventos políticos más importantes durante el año de análisis.
\end{abstract}

Palabras clave: Perú, Legislativo, gobierno dividido, vacancia, Odebrecht

\begin{abstract}
The popular Peruvian saying that "one suffers, but enjoys" summarizes the year 2017 nicely. The year saw numerous social, political, and economic difficulties in Peru. The incumbent government had to face the ravages of El Niño, ministerial crises, and vacancy requests, among other things, while at the same time the economy was slowing down and poverty increasing. However, we believe that for most Peruvians, 2017 was also a joyous year, especially considering the agonizing classification of the Peruvian team in advance of the World Cup after 36 years of absence. In this article, we summarize the social and political context of 2017, focusing on the executive-legislative axis that determined the most important political events during the year of analysis.
\end{abstract}

Keywords: Peru, Legislative, divided government, vacancy, Odebrecht 


\section{INTRODUCCIÓN}

En este artículo buscamos resumir lo acontecido en Perú durante 2017. Creemos que este tipo de empresas se enfrenta a dos imposibles: primero, cubrir todos los eventos relevantes, y al mismo tiempo, como segundo punto, evitar que nuestros sesgos profesionales determinen la centralidad del evento. Dicho esto, esperamos proveer de una narrativa que tenga dos finalidades: por un lado, brindar un resumen que permita al lector entender los hechos y debates centrales acontecidos, y, por otro lado, ayudarlo a entender los eventos subsiguientes.

El 2017 para Perú no fue un año sencillo. Fenómenos climatológicos afectaron gravemente toda la región norte del país y, al mismo tiempo, también la clasificación al Mundial de Fútbol fue un acontecimiento que estremeció a todos los fanáticos de la selección, tanto que la celebración del gol que selló la clasificación activó las alertas sísmicas. En lo político, el pasado 2017 fue sobre todo un año que no dio respiro a politólogos y analistas, en tanto se dictó prisión preventiva contra los expresidentes Alejandro Toledo y Ollanta Humala y a finales de año el gobierno otorgó el indulto humanitario a Alberto Fujimori. Todo esto en el marco de tensiones sin precedentes entre el Ejecutivo y Legislativo.

En la siguiente sección, resumimos la coyuntura social que se vivió en el país durante 2017, para luego centrarnos en los cambios institucionales y de políticas públicas promovidas por el gobierno de turno. Finalmente, nos concentramos en la relación entre el Ejecutivo y el Legislativo como eje para comprender los hechos políticos más importantes del año. Finalmente concluimos en la última sección.

\section{COYUNTURA SOCIAL}

El 2017 fue un año mezclado de noticias contrariadas. Por un lado, terminando el mes de enero y en el transcurso de los meses de verano, el país sufrió los efectos climatológicos del fenómeno del Niño, que debido a sus características fue denominado Niño Costero. El Niño Costero remarcó las precariedades y limitaciones del alcance del Estado peruano. En efecto, el ministro Fernando Zavala resumió los daños del Niño de la siguiente manera: 21.000 viviendas colapsadas, 20.000 inhabitables, 710 establecimientos de salud afectados y 39 colapsados, 2.000 locales escolares afectados, 3.000 kilómetros de la red vial nacional dañados, 323 puentes destruidos, 85.000 hectáreas de cultivo perdidas o afectadas, 184.000 damnificados y más de un millón de personas afectadas (Ballón 2017: 30-31). Por otro lado, a fines de 2017 y después de 36 años sin participar en un Campeonato Mundial de Fútbol, el Perú clasificó al de Rusia 2018. La clasificación fue muy larga y agónica, y terminó en un partido contra Nueva Zelanda por uno de los últimos cupos de la región de Sudamérica. La 
alegría de los partidos para la clasificación del mundial ocultaba temporalmente otros sucesos que acontecían en el país.

La migración venezolana hacia Perú también fue otra estampa distintiva del año. Miles de venezolanos decidieron migrar debido a las condiciones que se viven en su país. Si bien Perú no es el que ha recibido el mayor número de la diáspora venezolana, sí ha visto incrementar considerablemente el número de emigrantes de ese origen. El gobierno peruano decidió otorgar un Permiso Temporal de Permanencia (PTP) para los venezolanos que busquen trabajo formal. Según las cifras del gobierno peruano, más de 190 mil venezolanos y venezolanas ingresaron al país durante 2017 e inicios del 2018; sin embargo, solo 30 mil de ellos han solicitado un PTP. La inmigración de venezolanos en Perú ha concitado varias manifestaciones de solidaridad pero también algunas muestras de rechazo. Es un fenómeno nuevo para un país caracterizado por la emigración de connacionales y forma parte de una agenda pendiente entender cuáles son los cambios a largo plazo producto de este fenómeno.

A menos de seis meses del nuevo gobierno, los efectos del Niño Costero refutaban la imagen que el presidente Pedro Pablo Kuczysnki (PPK) quería proyectar al resto del país. ${ }^{1}$ Este fenómeno produjo impactos significativos sobre la actividad productiva y parecía que el gobierno no estaba preparado para afrontar sus consecuencias. PPK llegó al poder con grandes expectativas y con credenciales de un tecnócrata que mejoraría la economía, para lo que se rodeó de profesionales muy capacitados. En la prensa se hablaba de un "gabinete de lujo", ya que el mismo PPK hablaba así de los ministros que había elegido: "Es un gabinete de lujo, de la mejor gente de todo el Perú"(Correo 2016). Pero desde otros sectores, como el encarnado por el parlamentario opositor Víctor Andrés García Belaúnde, se lo calificaba de la siguiente manera: "Gobierno de lujo no, les gusta el lujo, practican el lujo, les encanta; todo lo que brilla para ellos es oro y el Gobierno, por supuesto, brilla muchísimo" (Correo 2016). El equipo de "lujo" de PPK estaba compuesto por personalidades que venían del sector privado empresarial, y muchos de ellos tenían una visión crítica del rol del Estado en la economía, sobre todo en el área de promoción del desarrollo.

Las relaciones Ejecutivo-Legislativo, como desarrollaremos más adelante, durante el gobierno de PPK fueron muy diferentes en comparación con gobiernos anteriores. El partido de gobierno, Peruanos por el Kambio, logró una representación muy reducida en el Congreso, mientras que el principal partido de oposición, Fuerza Popular, obtuvo una súper mayoría. Esto no había sucedido desde 2001. Y en el año y ocho meses que duró el gobierno de PPK, su equipo se limitó a continuar las políticas de gobiernos anteriores, y salvo algunas excepciones que discutiremos más adelante, en general no hubo grandes cambios.

Pedro Pablo Kuczynski es conocido como PPK. El nombre de su partido para las elecciones de 2016 fue Peruanos por el Kambio (PPK). Sobre las elecciones presidenciales, ver McNulty (2017). 


\section{CAMBIOS INSTITUCIONALES Y POLÍTICAS PÚBLICAS}

En los primeros cien días de gobierno, PPK buscó capitalizar su imagen de tecnócrata eficiente. El gobierno señalaba constantemente que su gestión impulsaría el "destrabe" de la inversión pública. Para esto, anunció el cambio del Sistema Nacional de Inversión Pública (SNIP) por otro supuestamente más ágil y menos burocrático. Además, en la evaluación de sus primeros cien días de trabajo, el gobierno listaba obras de envergadura como el reinicio de la construcción del metro de Lima, la construcción de miles de kilómetros de carretera, etc. De estos anuncios, resaltaba el Aeropuerto Internacional de Chincheros en Cusco, que le permitiría de manera fehaciente demonstrar la capacidad de destrabe de su gobierno. ${ }^{2}$ Finalizando 2016 e iniciando 2017, muchas de estas promesas no se materializaron, e incluso las negociaciones que buscaban iniciar la construcción del Aeropuerto de Chincheros le costaron el puesto al ministro de Transporte (Martín Vizcarra, ahora presidente de la República) y a varios altos funcionarios del Ministerio de Economía y Finanzas (MEF), además de protestas y huelgas en Cusco debido a las expectativas generadas. Destrabar no le resultó tan fácil como se pensaba.

La eficiencia de este gobierno no solo quedo en entredicho por la demora en los proyectos sino también por tener un menor desempeño que el gobierno de Humala (Meléndez y Sosa 2013; Barrenechea y Sosa 2014). Por ejemplo, si tomamos el dato de inversión pública como referencia, el gobierno de PPK, durante el 2017, logró ejecutar el 68\% del monto asignado a inversiones, en tanto que el gobierno anterior logró ejecutar el $71 \%$ de su presupuesto de inversión pública en un periodo similar (2012). En parte, la explicación de la lentitud o poca capacidad del gobierno recae en la inestabilidad política en la que estaba inmerso.

Desde que PPK empezó su gestión en julio de 2016, se han hecho 44 cambios en el Ejecutivo. Al realizar un balance de este año y ocho meses que duró el gobierno de PPK, esta cifra fue más alta que en tiempos de Ollanta Humala (41), Alan García (24) y Alejandro Toledo (27) (Perú21 2018). En otras palabras, en menos de dos años de gobierno, PPK tuvo más ministros que Ollanta Humala en cinco. En este contexto, es difícil pensar que las entradas y salidas del gabinete hayan podido conducir a grandes cambios en las políticas públicas.

La inestabilidad dentro del Ejecutivo amenazó los planes del gobierno en las áreas de educación, diversificación económica, y sobre todo no ayudó a revertir la desaceleración económica a consecuencia de la caída de los precios de las materias primas. Como se detalla más adelante, el primer ministro de Educación de PPK, Jaime Saavedra, tuvo que dejar su cargo en diciembre de 2016, aun teniendo altos índices de aprobación. El principal partido de oposición, Fuerza 
Popular, censuró a Saavedra debido a presuntos actos de corrupción. Según El Comercio, la destitución de su ministro más popular fue "un fuerte golpe para el gobierno de Pedro Pablo Kuczynski (PPK)" (BBC Mundo 2018). Después de Saavedra, tres ministros más salieron del gabinete tras acusaciones de corrupción o ineficiencia formuladas por el Congreso. Uno de ellos fue Martín Vizcarra, en ese entonces ministro de Transportes, cargo que ocupó hasta mayo de 2017. Le siguieron Alfredo Thorne, de Economía, Marilú Martens, de Educación, y junto con ella, casi todo el gabinete que funcionaba en el momento (BBC Mundo 2018).

Para algunos observadores, la salida rápida de ministros "de lujo" parecía indicar que ellos no estaban políticamente capacitados para enfrentar a un Congreso dominado por la oposición, es decir, Fuerza Popular, el partido de Keiko Fujimori. Incluso hubo otros que señalaban que la salida de Saavedra mostró debilidad de parte de PPK. Cualquiera de estas dos versiones confirma que las relaciones Ejecutivo-Legislativo durante el gobierno de PPK fueron muy diferentes de las de gobiernos anteriores, y terminarían con la renuncia de PPK en marzo de 2018.

En general, la política pública en los dos primeros años de gobierno de PPK no tuvo un norte definido; muchos de los programas iniciados en los gobiernos anteriores siguieron su rumbo sin mayor mejora o replanteamiento. En algunos casos, posiciones e instituciones claves como la SUNAT, la Defensoría del Pueblo, entre otras, se dejaron a merced del Legislativo o se nombraron funcionarios cercanos al partido de Fuerza Popular.

Durante la década del 2000, la economía peruana mantuvo un crecimiento considerable, principalmente a consecuencia de las exportaciones mineras (Arce 2005). Pero desde 2011 entró en un proceso largo de desaceleración. Y en el año 2014 se dio el crecimiento más bajo de la última década (2,4\%). Luego de un ligero repunte durante 2015 (4,0\%), el crecimiento del 2014 es muy similar al del año 2017 (2,5\%) (ver Gráfico 1). El menor crecimiento del PBI respondió a una desaceleración significativa del sector primario. Por ejemplo, el sector de minería metálica creció $4,2 \%$ en 2017, después de haber crecido $21,2 \%$ en 2016 (BCRP 2017: 17). La economía fue afectada por dos eventos de distintas magnitudes: el primero fue el Niño Costero mencionado anteriormente, que destruyó gran parte de la infraestructura del norte del país durante el primer trimestre de 2017; y el segundo fue el escándalo de corrupción del caso Lava Jato, que "afectó la confianza de los inversionistas y paralizó proyectos de inversión de asociaciones público-privadas" (BCRP 2017: 11). Por otro lado, en el 2017 la inflación anual fue de 1.36 por ciento, la tasa más baja desde el 2009 (BCRP 2017).

Las proyecciones macroeconómicas para 2018 confirman la desaceleración de la economía nacional. El Ministerio de Economía y Finanzas (MEF) inicialmente estimó un crecimiento del $4 \%$ para 2018. Ahora esta proyección se ha reducido al $3,6 \%$. Aunque el MEF anticipa que el sector minero tendría un mayor dinamismo por la mejora de los precios de los metales (MEF 2018), hay otras variables 
que confirman el deterioro del panorama económico. En 2017, por ejemplo, la presión tributaria alcanzó el 12,9 por ciento del PBI, y fue el nivel más bajo de los últimos 15 años (MEF 2018). El deterioro de las finanzas públicas motivó al gobierno actual de Vizcarra a imponer algunas medidas de austeridad fiscal y el incremento de algunos impuestos, lo cual provocó protestas y manifestaciones en diversas regiones del Perú.

La incidencia de la pobreza también nos permite apreciar el deterioro del panorama económico. En el 2017, el 21,7\% de los peruanos (casi 7 millones de personas) se registraron en condición de pobreza (Gráfico 2). La cifra de 2017 representa un incremento del 1 por ciento con respecto a la de 2016, y equivale a 375 mil nuevos pobres para ese año. También cabe resaltar que este aumento es el primer incremento que se observa desde 2007, cuando la incidencia de pobreza fue del 42,4 por ciento (MEF 2018). El incremento este año afectó principalmente a la zona rural, cuya tasa de pobreza es tres veces mayor que la urbana (44,4\% y 15,1\% respectivamente). En Lima Metropolitana, la pobreza afectó al 13,3\% de la población, es decir, 2,3 puntos más que en el año 2016 (INEI $2018 b$ ). Por otro lado, la incidencia de pobreza extrema afectó al 3,8 por ciento de la población del país y no mostró variación con la cifra de 2016 (Gráfico 2). Como es conocido, la Sierra registra la mayor pobreza extrema, afectando al $8 \%$ de su población, seguida de la Selva con 6,2\% (INEI 2018a).

Como señalan Muñoz y Guibert (2016: 319), durante el ciclo electoral de 2016 el tema que varios candidatos presidenciales abordaban no era el cuestionamiento al modelo económico per se, sino las limitaciones del modelo basado centralmente en la exportación de minerales, en un contexto en el cual el precio de los commodities está en caída. En un principio, tanto Humala como PPK hablaban de la necesidad de destrabar las grandes inversiones mineras que estaban obstaculizadas por la oposición social (Arce 2014), en un escenario, además, de fuerte corrupción y polarización. El ministro de Economía y Finanzas de PPK, Alfredo Thorne, estimaba el valor de estas inversiones trabadas en 18.000 millones de dólares, lo cual podría tener un efecto fuerte en la expansión de la inversión privada y pública (Ballón 2017: 32). Pero estos proyectos, como Conga en Cajamarca y Tía María en Arequipa, no avanzaron a pesar del interés del gobierno y de los grandes inversionistas nacionales como internacionales en que lo hicieran.

Paralelamente, otros analistas hablaban de la necesidad de diversificar la economía nacional. En el gobierno de Humala, en particular, se planteó la la participación del Estado en actividades promotoras de la economía. Se crearon, por ejemplo, los Centros de Innovación Tecnológica (CITE) para apoyar técnicamente a los micro y pequeños productores. Y salieron normas con beneficios tributarios al gasto de investigación y desarrollo, que entraron en vigencia en 2016. Pero el gobierno de PPK se inclinó a favor de quienes proponían una posición más ortodoxa en términos económicos. Para ellos la diversificación productiva debe ser decidida por los empresarios y el mercado, y no por el Estado. Otros ministros de PPK también se manifestaban en contra 
de la participación del Estado en actividades promotoras de la economía. Y el ministro de Economía y Finanzas Thorne buscaba agilizar, desburocratizar y destrabar todo tipo de proyectos. Finalmente, PPK tuvo tres ministros en la cartera de Economía en menos de dos años de gobierno, y para mucho estos fracasaron en reactivar la economía (ver Gráfico 1). El incremento de la incidencia de la pobreza en 2017 también hace cuestionar la gestión gubernamental del supuesto "gabinete de lujo" convocado por PPK (ver Gráfico 2). El gobierno de Martín Vizcarra, sin embargo, parece haber retomado la importancia de la diversificación productiva, proceso que otros denominan "dirigismo" (Gestión 2016).

Grafico 1: Variación anual del PBI real (\%) 2001-2017

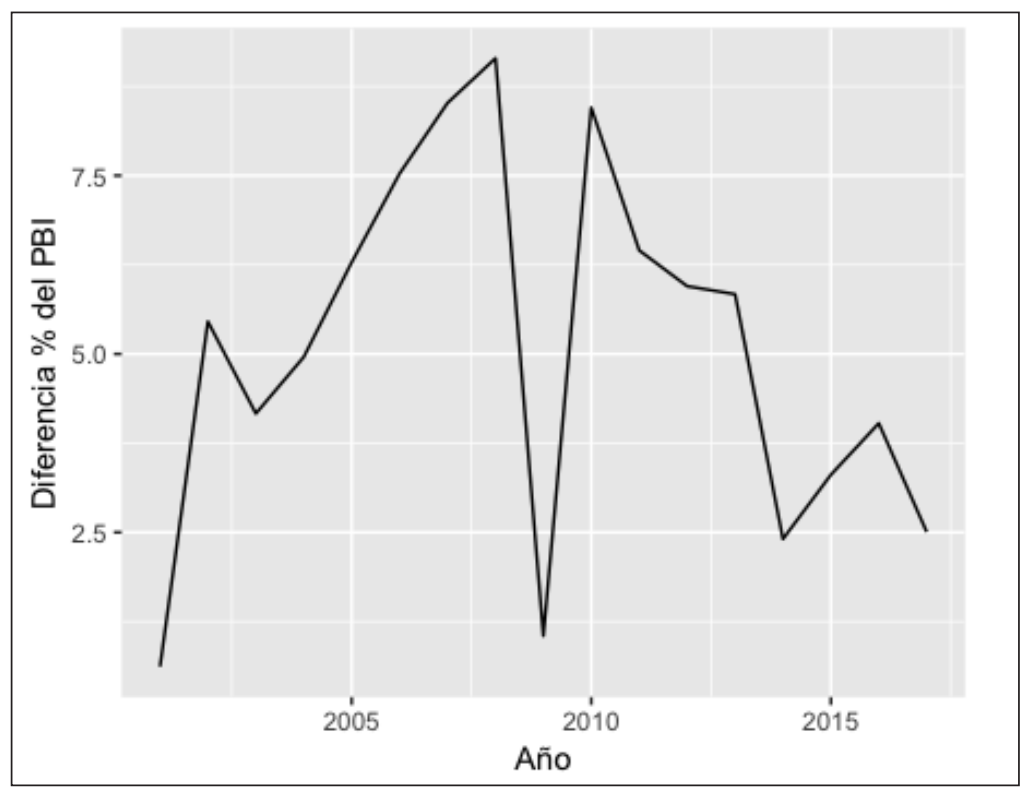

Fuente: Elaboración propia a base de datos del BCRP 
Gráfico 2: Incidencia de la pobreza total y extrema 2009-2016

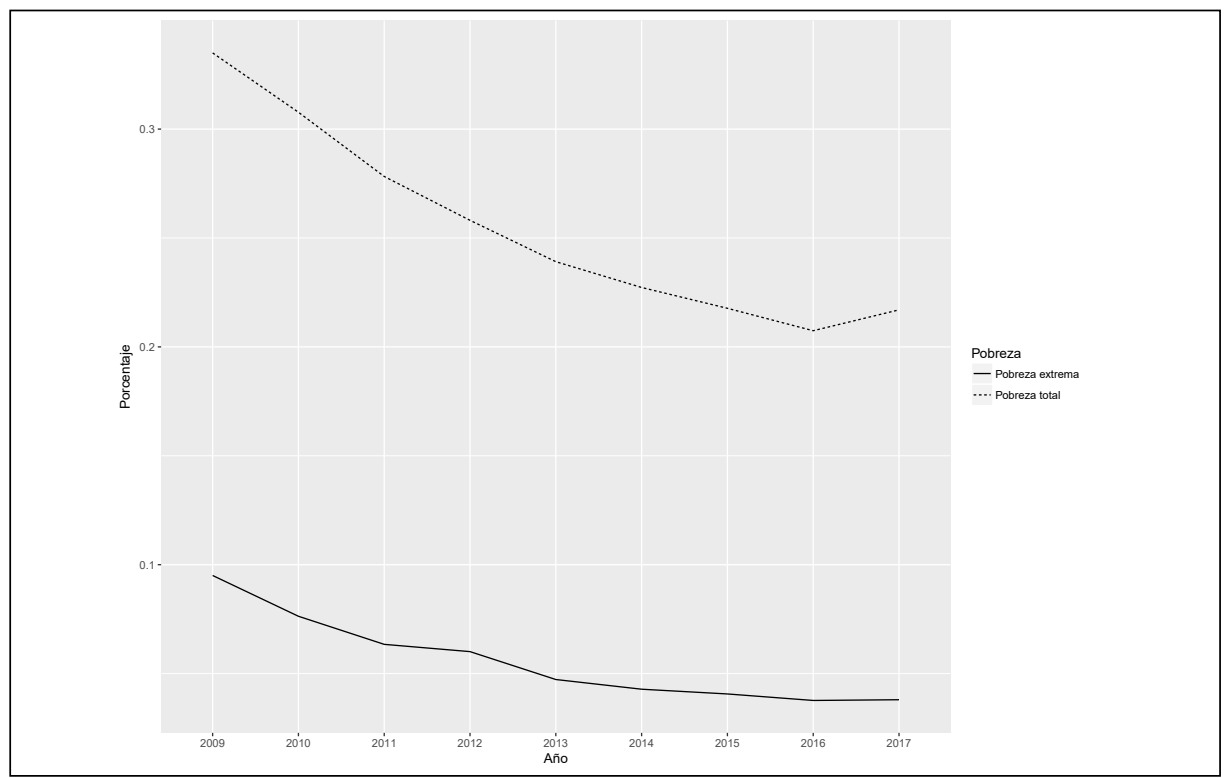

Fuente: Elaboración propia a base de datos del INEI

\section{PODER EJECUTIVO Y PODER LEGISLATIVO}

Desde el retorno a un régimen democrático en 2001, las relaciones entre el Ejecutivo y el Legislativo no han sido fáciles. La tensión entre el partido que controla el Ejecutivo y la representación congresal ha estado marcada por disputas mediáticas y constantes intentos de imposición de un poder sobre el otro. Sin embargo, durante 2017 los niveles de tensión y conflicto entre ambos alcanzaron niveles sin precedentes desde el retorno a la institucionalidad democrática. El epítome de este enfrentamiento se dio a inicios de 2018, donde el presidente electo se vio forzado a renunciar. La incertidumbre política agrava el descontento e incredulidad de la ciudadanía con el régimen democrático.

Los resultados electorales de 2016 nos enfrentaron con una situación sin paralelo, donde el principal partido de oposición, Fuerza Popular, logró obtener una súper mayoría en el Congreso, en tanto que la representación del partido de gobierno, Peruanos por el Kambio, logró una reducida representación. Perú es un sistema semipresidencial, donde el Congreso está facultado para remover ministros, declarar la vacancia presidencial y nombrar funcionarios clave, entre otros. Desde 2001 que, si bien el partido oficialista no obtenía mayoría, era la primera minoría, logrando ser un poder de veto importante en las actividades del Congreso. Esto cambió radicalmente con el gobierno de Pedro Pablo Kuczynski. En el gráfico 3 se muestra el porcentaje del Congreso controlado por el partido de gobierno y la principal fuerza opositora en los últimos cuatro períodos. 
Gráfico 3. Correlación de fuerzas entre oficialismo y oposición en el Legislativo peruano 2001-2016

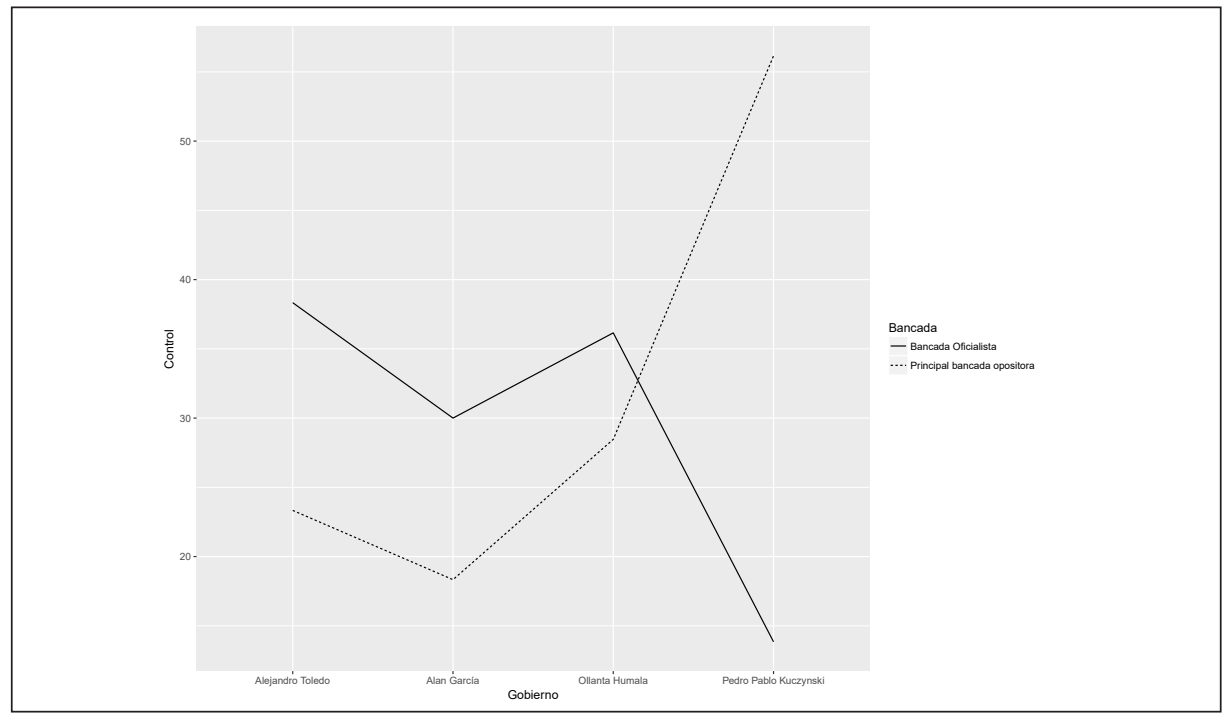

Fuente: Elaboración propia a base de datos INFOGOB

Desde Toledo (2001) hasta Humala (2011), el Ejecutivo se iniciaba con un número importante de congresistas. Toledo y Humala comenzaron su gestión como la primera minoría mientras que el gobierno de García (2006-2011) se convirtió en primera minoría luego de que la principal bancada opositora se dividiera durante su primer año. En resumen, los tres primeros gobiernos democráticos contaban con una fuerza importante en el Legislativo, lo que les permitía negociar, proponer agenda e incluso controlar a la oposición y, en la mayoría de los casos, podían imponerse en la elección de la mesa directiva (Gráfico 3). La situación del gobierno de PPK era extremadamente precaria, lo cual fue aprovechado por la bancada fujimorista, que impuso agenda y logró imponer el poder Legislativo sobre el Ejecutivo.

Tanto elCongreso y como el Ejecutivo son conformados en elecciones democráticas, por lo que ambos hasta cierto punto se pueden irrogar el mandato popular, esto es lo que Linz (1998) señalaba como el problema de la "doble legitimidad" en el presidencialismo. Esto se hizo evidente al conocerse los resultados de las elecciones y la composición del Congreso, cuando la lideresa de Fuerza Popular, Keiko Fujimori, dio una conferencia de prensa donde aseguró a sus electores que se cumplirían las promesas hechas durante la campaña. En estas declaraciones la acompañó toda su bancada, mostrando que tenía todo el poder para hacerlo.

En resumen, Kuczynski tenía que lidiar con un gobierno en extremo dividido, donde como veremos más adelante la tensión se fue incrementando durante el año, siendo el punto más álgido la fallida vacancia presidencial, el indulto a Alberto Fujimori y la disputa por el liderazgo del fujimorismo. 


\section{Correlación de fuerzas, las primeras víctimas}

La fortaleza de Fuerza Popular en el Congreso no es gratuita. La construcción del partido se debe a un arduo trabajo de captación y fortalecimiento de cuadros, pero sobre todo a una inicial decisión de Keiko Fujimori al optar por la construcción partidaria. Es importante recalcar esta opción, ya que rompe con la tradición fujimorista de basar la política en el líder sin la necesidad de fortalecer o formar partido político. ${ }^{3}$

En un primer momento, Keiko Fujimori capitalizó la popularidad de su padre, y en su primera candidatura política logró ser electa congresista con la votación preferencial más alta del Congreso, lo que le permitió consolidar su liderazgo frente a otras figuras del fujimorismo. Este resultado demostró que pese a la reciente caída del régimen fujimorista y al encarcelamiento de Alberto Fujimori, como movimiento político seguía vigente y era un espacio por aprovecharse. Keiko Fujimori fundó Fuerza Popular, ${ }^{4}$ con la finalidad de participar en las elecciones presidenciales de 2011. Su estrategia radicó en ampliar sus bases partidarias a lo largo del país, añadiendo cuadros en las diferentes circunscripciones subnacionales. En la Tabla 1 se muestra la participación de Fuerza Popular en las elecciones subnacionales.

Tabla 1. Participación de Fuerza Popular en las elecciones subnacionales previas a la elección presidencial 2016

\begin{tabular}{lcccccc}
\cline { 2 - 7 } & \multicolumn{2}{c}{ Distrital (1639) } & \multicolumn{2}{c}{ Provincial (195) } & \multicolumn{2}{c}{ Regional (25) } \\
\cline { 2 - 7 } & 2010 & 2014 & 2010 & 2014 & 2010 & 2014 \\
\hline Participación & $27.94 \%$ & $43.47 \%$ & $35.38 \%$ & $53.33 \%$ & $40 \%$ & $64 \%$ \\
Ganadas & $11.57 \%$ & $10.47 \%$ & $7.25 \%$ & $3.85 \%$ & $0 \%$ & $0 \%$ \\
Autoridades Electas & 315 & 474 & 50 & 68 & 5 & 5 \\
\hline
\end{tabular}

Fuente: Elaboración propia a base de datos INFOGOB

Resaltamos que el crecimiento de las bases políticas se ve reflejado en la capacidad del partido de poder presentar candidatos en las distintas circunscripciones del país. Como se puede apreciar, entre las elecciones subnacionales de 2010 y 2014 se evidencia un aumento importante en la participación de sus candidatos. El absoluto de circunscripciones ganadas también aumentó; sin embargo, el porcentaje no refleja este aumento, ya que el universo de circunscripciones en las que participó también se incrementa. 
Este constante posicionamiento de Fuerza Popular en los niveles subnacionales le permitía mantener una extendida presencia que repercutía en las elecciones nacionales. Así, en los siguientes comicios (2011 y 2016), Keiko Fujimori llegó a estar cerca de obtener la elección presidencial. En cada balotaje, el competidor de turno pudo aglomerar el voto "antifujimorista", en este caso apoyado por organizaciones civiles que implementaron importantes campañas en contra de Keiko y contribuyeron a su derrota. Estos colectivos tuvieron un importante rol en la última campaña presidencial.

El caso de Pedro Pablo Kuczynski es el opuesto al de Fuerza Popular. PPK se presentó a las elecciones de 2011 en una alianza multipartidaria sin mayor coherencia ideológica o programática. ${ }^{5}$ El ascenso de PPK como candidato durante la última elección se puede explicar por las circunstancias de la competencia electoral, donde el candidato con mayores chances de pasar a la segunda elección presidencial, Julio Guzmán, fue retirado de la contienda por el Jurado Nacional de Elecciones. Esto dejó libre el segundo puesto, para el que los candidatos disponibles eran Verónica Mendoza y Pedro Pablo Kuczynski. PPK logró capitalizar el voto de los sectores de clase media y de derecha que veían a Verónica Mendoza como una amenaza para el sistema económico. En resumen, el ascenso de PPK y su posterior victoria no se dio por mérito propio sino como una conjunción de eventos coyunturales y la activa participación de un movimiento ciudadano sin mayor eje de cohesión que el voto antifujimorista. El costo de la improvisación se vio reflejado en la disminuida presencia del partido de gobierno en el Congreso, donde no podía hacer frente a la mayoría fujimorista.

La primera víctima de la disparidad de fuerzas entre el Ejecutivo y Legislativo fue el ministro de Educación, Jaime Saavedra. Ocupó el mismo cargo durante el gobierno de Ollanta Humala, y en una señal positiva, PPK lo invitó a continuar durante su gobierno. La figura de Jaime Saavedra no era del agrado del fujimorismo, en parte porque su permanencia en el gobierno representaba el éxito de una política pública gestionada en la gestión de Humala.

La remoción de Saavedra en octubre de 2016 fue un punto de quiebre en el equilibrio entre poderes, ya que esa disputa le mostró al Legislativo que el Ejecutivo no tenía mayor capacidad de reacción. Este último inicialmente amenazó con usar la prerrogativa constitucional de solicitar la confianza a todo el gabinete si es fujimorismo seguía con su intento de remover a Saavedra. Dicha solicitud de confianza abría la posibilidad de permitir al Ejecutivo cerrar el Congreso y llamar a elecciones anticipadas del Poder Legislativo. El gobierno se debatía entre pedir o no la "cuestión de confianza", optando finalmente por no hacerlo. El Legislativo removió al ministro de Educación, y el gobierno perdió credibilidad en el uso de su prerrogativa constitucional de cerrar el Congreso.

Pedro Pablo Kuczynski postuló como candidato de una alianza integrada por un partido de derecha (Partido Popular Cristiano), de izquierda (Partido Humanista), evangélico (Movimiento Restauración Nacional) y un partido regionalista (Alianza por el Progreso). La alianza no duró más allá de la primera elección presidencial. Ver El Comercio (2017b). 
El Legislativo debilitaba al Ejecutivo aprovechando su prerrogativa de interpelación y censura a ministros de Estado. Durante 2017 ya sea por presión o por remoción directa del Congreso el gobierno realizó cuatro cambios ministeriales. ${ }^{6}$

En agosto de 2017, el Legislativo aprobó una moción de interpelación contra la ministra de Educación, Marilú Martens. En septiembre, el Congreso decidió presentar un pedido de vacancia contra la ministra y, como consecuencia, el presidente del Consejo de Ministros, Jaime Zavala, decidió pedir la "cuestión de confianza", activando el procedimiento que le permitiría al presidente cerrar el Congreso y llamar a elecciones anticipadas. Este tomó el reto y negó la confianza, dando paso a la primera crisis ministerial total del gobierno, que tuvo dos opciones: hacer creíble su amenaza nombrando a un gabinete similar al anterior y mandando una señal de resistencia, o nombrar un gabinete que fuera del beneplácito de la mayoría fujimorista. Optó por lo segundo y, Mercedes Aráoz, la nueva presidenta del Consejo de Ministros, eligió funcionarios cercanos al fujimorismo o a sus aliados (El País 2017), con la esperanza de lograr un periodo de calma para su gobierno.

\section{Escándalo Lava Jato}

Los escándalos de corrupción que involucran a la constructora Odebrecht en la compra de funcionarios y políticos en Perú marcaron el año 2017, pues la población semana tras semana se enteraba de pedidos de detención contra altos funcionarios de gobiernos anteriores por parte de la fiscalía.

En este contexto, los actores judiciales concentraron notoriedad durante 2017. Los peruanos fuimos testigos de la inclusión en calidad de investigados de expresidentes y altos funcionarios de gobiernos previos. Durante el año, presenciamos el encarcelamiento de exviceministros, así también de algunos funcionarios y directivos de las empresas involucrados se acogieron como colaboradores eficaces (El Comercio 2017a). Los medios de comunicación cubrían los allanamientos de residencias y oficinas de los involucrados, muy cerca del estilo de una serie producida en Hollywood. De pronto, nombres de jueces y fiscales se volvieron $\operatorname{conocidos}^{7}$ por la opinión pública. Las columnas de opinión recogían una sensación compartida de inusual confianza en los sistemas de justicia, y se vivió momentos de optimismo en la lucha contra la corrupción. Sin embargo, con el transcurso del año, también se hizo evidente que no todos los fiscales tenían la celeridad o arrojo, creando la percepción de que algunos implicados eran tratados con más dureza que otros. ${ }^{8}$ 
El trabajo de fiscales y jueces, sobre todo con casos de altos funcionarios y políticos, fue una postal representativa de 2017. Así, en agosto, el expresidente Ollanta Humala y su esposa Nadine Heredia ingresaban a prisión preventiva solicitada por el fiscal que investiga el caso de los aportes de la constructora brasileña durante la campaña de 2016. Por otro lado, la fiscalía solicitó la captura internacional del presidente Alejandro Toledo, quien reside en los Estados Unidos, y mientras se escribe este artículo se procesa su extradición.

El escándalo Lava Jato no solo afecto a expresidentes sino que también fue el centro de la crisis del gobierno de PPK. La corta calma después del nombramiento de Mercedes Aráoz como presidenta del Consejo de Ministros no duró mucho. En medio de las tensiones entre Legislativo y Ejecutivo, la comisión del Congreso que investigaba los hechos de corrupción en el escándalo Lava Jato, controlada por Fuerza Popular, empezó a indagar la relación de PPK con la constructora Odebrecht y su involucramiento en los casos de compra de funcionarios por parte de la constructora brasileña.

El 13 diciembre la presidenta de la comisión, Rosa Bartra, mostró información que relacionaba la consultora de PPK con pagos de la constructora cuando era ministro de Alejandro Toledo (2001-2006). Este hecho significó la oportunidad para que la mayoría fujimorista promoviera la vacancia del presidente por incapacidad moral. El involucramiento de PPK en el escándalo Odebrecht confirmaba la percepción de que toda la clase política estaba involucrada en el esquema de corrupción promovido por la empresa brasileña.

El grupo parlamentario de izquierda Frente Amplio pidió la vacancia presidencial. La solicitud dividió al Congreso y a los partidos. Dentro del partido Aprista, por ejemplo, no había un consenso sobre si se debía vacar o no al presidente. La bancada de Nuevo Perú, también de izquierda, dudaba en apoyar la vacancia presidencial por el temor de afectar la institucionalidad entregando el Ejecutivo al fujimorismo. ${ }^{9}$ Sumando los votos del Frente Amplio con los del fujimorismo la vacancia contaba con 81 votos y eran necesarios 87 para vacar al presidente. La bancada oficialista contaba con 18 votos. Por su parte, los demás grupos parlamentarios no dieron conocer su voto o mandaron señales ambiguas sobre él.

El 20 de diciembre se votó el pedido de vacancia presidencial, lo que mantuvo al país en un tenso suspenso. La defensa del presidente Kuczynski frente al Congreso tomó 11 horas, la votación final estaba rodeada de un velo de incertidumbre. Esta mostró varias sorpresas: por ejemplo, bancadas caracterizadas por su disciplina partidaria, como la Aprista, dividieron sus votos. La bancada de Nuevo Perú se retiró en el preciso instante de la votación. Sin embargo, el golpe final contra la vacancia vino del propio fujimorismo, donde Kenji Fujimori (hermano menor de Keiko Fujimori) convenció a diez legisladores de Fuerza Popular y logró que

9 En este contexto los vicepresidentes habían anunciado que de votarse la vacancia renunciarían, por lo que el presidente del Congreso asumía la presidencia y llamaba a elecciones generales. 
se abstuvieran en la votación. Este inesperado golpe tomó a todos por sorpresa y reveló que la unidad del partido y el liderazgo de Keiko Fujimori estaban siendo amenazados.

Kuczynski no fue vacado en esta oportunidad, pues la opción obtuvo 79 votos (faltaron 8 necesarios), lo que hizo que la abstención de la facción de Kenji Fujimori fuera mucho más relevante. PPK logró reducir la fuerza fujimorista y darle un respiro a su gobierno. Sin embargo, el favor del menor de los Fujimori tuvo un alto precio: el indulto de Alberto Fujimori.

\section{La Navidad de los Fujimori}

La noche del 24 de diciembre, a pocos días del intento de vacancia presidencial, Kuczynski otorgó el indulto humanitario a Alberto Fujimori, quien estaba purgando condena por violación de los derechos humanos. La noticia fue el centro de discusión en el país; los colectivos, movimientos y partidos de izquierda empezaron a organizar inmediatamente movilizaciones y demostraciones de protesta. Las acciones empezaron esa misma noche, y PPK tuvo que salir a dar un mensaje improvisado a través de redes sociales justificando la medida. Durante los siguientes días, Kuczynski y su primera ministra Mercedes Aráoz negaron que el indulto hubiese sido negociado como un intercambio por la vacancia. Sin embargo, indagaciones periodísticas mostraban innumerables vicios de forma y fondo en el otorgamiento del indulto.

Durante la campaña electoral, Kuczynski se comprometió a no indultar a Alberto Fujimori. El objetivo de esta promesa era captar a sectores de izquierda y de clase media progresista para poder revertir la ventaja que le llevara Keiko Fujimori. Este compromiso logró convencer a Verónica Mendoza de endosar sus simpatizantes para que votaran por Kuczynski. El indulto otorgado la noche previa a las celebraciones de Navidad enfureció y movilizó a estos sectores de inmediato, pues mostraban su desacuerdo y frustración al sentirse engañados.

La salida de Alberto Fujimori de la cárcel fue ampliamente cubierta por los medios de comunicación. La presencia de Alberto reforzó la presencia de Kenji Fujimori, que empezó a capitalizar la figura de su padre, el fortalecimiento de Kenji Fujimori debilitaba el liderazgo de su hermana, en tanto no podía criticar abiertamente el indulto a su padre, aun cuando este afectaba su liderazgo en favor de su hermano. Las siguientes semanas, el acercamiento entre Kuczynski y Kenji Fujimori se hizo más evidente, y este último daba a entender que podía postular a la presidencia en 2021 y que su "grupo" parlamentario seguiría creciendo con otras disidencias de Fuerza Popular.

El 2017 se acabó dejando un velo de incertidumbre muy grande para 2018. Por un lado, el Ejecutivo se encontraba en una situación de extrema debilidad, donde la ya pequeña bancada del partido oficialista perdió tres congresistas porque renunciaron a la bancada a modo de protesta por el indulto concedido a Alberto 
Fujimori. En el bando contrario, el fujimorismo enfrentaba un sisma interno y la figura de Keiko Fujimori perdía fuerza frente a su hermano. En tanto, entre los ciudadanos, la desaprobación de la élite política aumentaba y la parálisis política detenía los procesos de reforma educativa y la reconstrucción de la zona norte del Perú, fuertemente afectada por los fenómenos naturales de inicios del año. Los eventos del 2017 afectaron la aprobación tanto Kuczynski como de Keiko Fujimori, que poco pudieron hacer para evitar el deterioro constante de su aprobación ciudadana. (ver Gráfico 4).

\section{Gráfico 4: Aprobación de PPK y Keiko Fujimori}

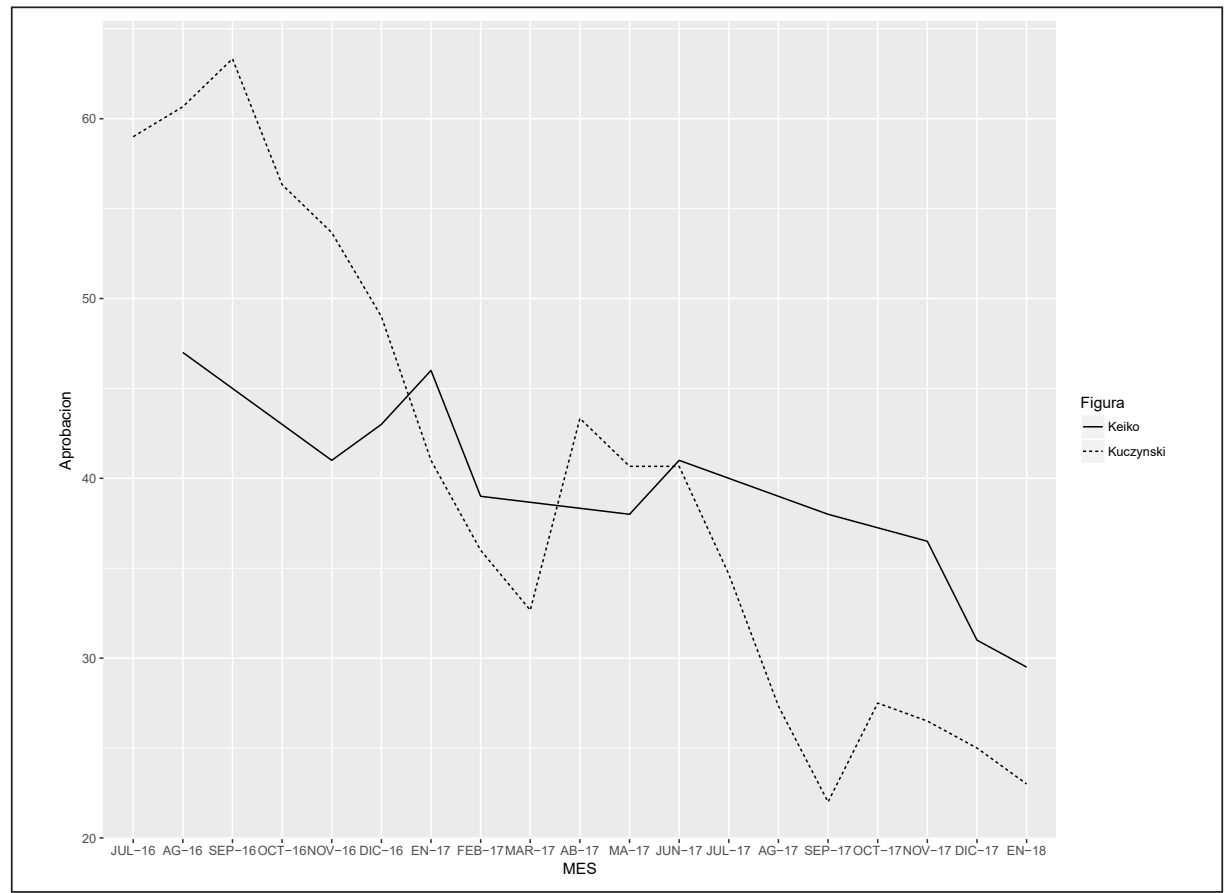

Fuente: Elaboración propia con base en reportes de IPSOS-Perú s.f. y GFK-Perú s.f.

Si bien en este artículo nos debemos ceñir a los eventos de 2017, creemos que al menos debemos resumir lo acontecido en los primeros meses de 2018. Kuczynski calculó mal, el indulto no solo movilizó grupos de derechos humanos sino que también le generó la pérdida de congresistas de su bancada y el apoyo de otros partidos, quienes empezaron a promover la idea de una nueva vacancia presidencial.

A inicios de marzo del 2018, los representantes de Alianza por el Progreso, partido que en su mayoría se abstuvo de votar en el primer intento de vacancia, promovieron una nueva solicitud. Mientras tanto, en el fujimorismo, Kenji Fujimori siguió en el proceso de captación de más congresistas. Frente 
al nuevo intento de vacancia, el gobierno de Kuczynski esperó que el sisma fujimorista se agravara y que la posición de Kenji Fujimori se reforzara. El desenlace fue tan sorpresivo como la votación de diciembre de 2017. Uno de los congresistas contactado por el grupo de Kenji Fujimori, Moises Mamani, grabó las conversaciones donde se le ofrecían contratos de inversión pública y obras a cambio de su voto en contra de la vacancia. Este nuevo escándalo a días de la votación forzó la renuncia de Kuczynski el 21 de marzo. El vicepresidente Vizcarra asumió la presidencia de la república y Keiko Fujimori recuperó el liderazgo indiscutible de Fuerza popular, al menos por ahora.

\section{CONCLUSIONES}

Al inicio de su gobierno PPK prometió que Perú ingresaría a la Organización para la Cooperación y el Desarrollo Económico (OCDE). La realidad no tardaría en demostrarle que los problemas que enfrentaba eran más complejos de lo que esperaba. No sabremos si recordó esa promesa mientras firmaba su renuncia a la presidencia a menos de dos años de haber ganado las elecciones generales.

Perú enfrenta para este y los siguientes años importantes retos. Después de casi dos décadas de crecimiento ininterrumpido, no se ha podido combatir la informalidad o erradicar la pobreza. Incluso según un reporte del Instituto Nacional de Estadística e Informática (INEI) la pobreza en 2017 aumentó por primera vez en 11 años (INEI 2018a).

El diseño constitucional y el orden democrático también han sido puestos a prueba en 2017. Los partidos enfrentan el reto de fortalecer su representación y buscar una consolidación democrática. Perú se acerca al bicentenario de su independencia con un gobierno débil y un sistema político fragmentado y poco representativo. La falta de institucionalidad política y la creciente desigualdad otorgan condiciones para que propuestas populistas lleguen al poder.

La reforma política es un debate constante y es una propuesta común cuando se buscan soluciones. Sin embargo, esta pierde fuerza y sentido cuando lo único que se logra aprobar son cambios de forma sin mayor impacto. No existe un debate serio acerca del objetivo del sistema político, en el que los partidos con representación nacional buscan cerrar el sistema a nuevos actores.

En resumen, 2017 es producto de un creciente deterioro del sistema político y las expectativas para 2018 y los años siguientes no son promisorias, a menos exista una voluntad de reconstruir y reconectar la representación política con la ciudadanía, evitando que esta última se termine de desencantar con el sistema democrático. 


\section{REFERENCIAS}

Arce, Moisés. 2005. Market Reform in Society: Post-crisis Politics and Economic Change in Authoritarian Peru. University Park: Pennsylvania State University Press.

Arce, Moisés. 2014. Resource Extraction and Protest in Peru. Pittsburgh: University of Pittsburgh Press.

Ballón, Eduardo. 2017. "Como elefantes en cristalería." En Perú Hoy: El arte del desgobierno, editado por Eduardo Toche Medrano. Lima: DESCO, 23-39.

Banco Central de Reserva del Perú (BCRP). 2017. Memoria 2017. Lima: Banco Central de Reserva del Perú.

Barrenechea, Rodrigo y Paolo Sosa. 2014. "Perú 2013: La paradoja de la estabilidad." Revista de Ciencia Política 34 (1): 267-292.

BBC Mundo. 2018, 23 de marzo. “Cómo el 'gobierno de lujo' de PPK en Perú acabó tan estrepitosamente y en menos de dos años."

Correo. 2016, 17 de julio. "Kuczynski: 'Gabinete es de lujo y tiene a la mejor gente del Perú'."

El Comercio. 2017a. "Odebrecht: Edwin Luyo evalúa acogerse a la colaboración eficaz."

El Comercio. 2017b. "Alianza de PPK se desintegra: Simon, Acuña y Lay formarán bancada."

El País. 2017, 18 de septiembre. "El presidente de Perú nombra nuevos ministros y cede ante la oposición."

GFK- Perú. s.f. https:/ /www.gfk.com/es-pe/

Gestión. 2016, 8 de agosto. "Giuffra: La diversificación productiva lo hacen los empresarios, no soy amigo del 'dirigismo'."

Instituto Nacional de Estadística e Informática (INEI). 2018a. Evolución de la Pobreza Monetaria 2007-2017: Informe Técnico. Lima: Instituto Nacional de Estadística e Informática.

Instituto Nacional de Estadística e Informática (INEI). 2018b. "Pobreza monetaria afectó al 21.7 por ciento de la población del país durante el año 2017." Lima: Instituto Nacional de Estadística e Informática, Nota de Prensa, 24 de abril. Recuperado el 9 de junio del 2018 de https: / /www.inei.gob.pe/

IPSOS-Perú. s.f. https:/ / www.ipsos.com/es-pe/news-and-polls/overview

La República. 2018, 19 de marzo. "Carlicantura."

Meléndez, Carlos y Sosa, Paolo. 2013. "Perú 2012: ¿Atrapados por la historia?" Revista de Ciencia Politica 33 (1): 325-350.

Ministerio de Economía y Finanzas (MEF). 2018. “Informe de Actualización de Proyecciones Macroeconómicas 2018-2021." Lima: Ministerio de Economía y Finanzas, 27 de abril. Recuperado el 9 de junio del 2018 de https:/ / www.mef.gob.pe/es /

McNulty, Stephanie. 2017. "Peru 2016: Continuity and Change in an Electoral Year." Revista de Ciencia Política 37 (1): 563-587.

Muñoz, Paula y Yamilé Guibert. 2016. "Perú: El fin del optimismo." Revista de Ciencia Política 36 (1): 313-338.

Perú21. 2018, 9 de mayo. "Pedro Pablo Kuczynski y sus ministros: del gabinete 'de lujo' al gabinete 'de lejos.'"

Moisés Arce es profesor de ciencia política de la Universidad de Missouri. Es autor de La extracción de recursos naturales y la protesta social en el Perú (PUCP, 2015). Sus temas de interés son la política cotenciosa, economía política y opinión pública, entre otros. Email: arcem@missouri.edu

José Incio es estudiante en el programa de doctorado en ciencia política de la Universidad de Pittsburgh, licenciado en ciencia política por la Pontifícia Universidad Católica del Perú. Sus temas de interés son política subnacional, desigualdad y política, entre otros. Email: j.incio@pitt.edu 